\title{
Effect of isoflurane alone or in combination with meloxicam on the behavior and physiology of goat kids following cautery disbudding
}

\author{
Melissa N. Hempstead, ${ }^{*} \dagger$ Joseph R. Waas, $†$ Mairi Stewart, $\ddagger$ Suzanne K. Dowling, ${ }^{\star}$ Vanessa M. Cave, \\ Gemma L. Lowe, ${ }^{*} \ddagger$ and Mhairi A. Sutherland*1 \\ *AgResearch Ltd., Ruakura Research Centre, Private Bag 3123, Hamilton, 3240, New Zealand \\ †School of Science, The University of Waikato, Private Bag 3105, Hamilton, 3240, New Zealand \\ łlnterAg, Ruakura Research Centre, Hamilton, 3214, New Zealand
}

\section{ABSTRACT}

Cautery disbudding of goat kids is painful, but may be alleviated with pain mitigation. We therefore evaluated the effect of administering general anesthesia (isoflurane) or a nonsteroidal anti-inflammatory drug (meloxicam) on goat kid behavior and physiology following cautery disbudding. Trial 1 ( $\mathrm{n}=12$ / treatment) evaluated behavioral responses in 72 female Saanen dairy goat kids (mean \pm standard error of the mean; $3.9 \pm 0.15 \mathrm{~d}$ old $)$ and trial $2(\mathrm{n}=10 /$ treatment) evaluated physiological responses in 60 female Saanen dairy goat kids $(4.3 \pm 0.14 \mathrm{~d}$ old $)$. Goat kids were randomly assigned to 1 of 6 treatment groups that were either (1) sham-handled only (simulated disbudding; SHAM) or disbudded with (2) no pain relief (CAUT), (3) isoflurane gas (ISO), (4) isoflurane and s.c. meloxicam combined (ISO+MEL), (5) meloxicam s.c. $(0.5 \mathrm{mg} / \mathrm{kg}$ of body weight; I-MEL), or (6) oral meloxicam $(0.2 \mathrm{mg} / \mathrm{kg}$ of body weight; O-MEL). Head shaking, head scratching, self-grooming, feeding, and body shaking were continuously video recorded for $24 \mathrm{~h}$ pre- and post-treatment. Lying behavior was recorded continuously for $24 \mathrm{~h}$ pre- and post-treatment using accelerometers. Plasma cortisol, glucose, and lactate concentrations were measured from blood samples collected immediately before treatment (baseline) and at 15,60 , and 120 min post-treatment. Body temperature was measured immediately after blood sampling at all blood sampling time points. Head shaking and body shaking frequencies were $50 \%$ higher in CAUT than SHAM kids 5 min post-treatment; ISO+MEL and ISO kids performed $25 \%$ less body shakes than CAUT kids. Head scratching durations $1 \mathrm{~h}$ post-treatment were higher in CAUT than SHAM kids, whereas O-MEL were similar to SHAM kids from $2 \mathrm{~h}$ post-treatment.

Received July 16, 2017.

Accepted December 6, 2017.

${ }^{1}$ Corresponding author: Mhairi.Sutherland@agresearch.co.nz
Self-grooming, feeding, and lying did not differ between groups. Cortisol concentrations were higher in CAUT than SHAM kids $(156.4 \pm 26.41$ and $104.1 \pm 26.41$ $\mathrm{nmol} / \mathrm{L}$, respectively), whereas ISO+MEL and ISO kids $(88.3 \pm 26.41$ and $113.2 \pm 26.41 \mathrm{nmol} / \mathrm{L}$, respectively $)$ had lower cortisol concentrations than CAUT kids over the 2-h sampling period. Moreover, O-MEL and I-MEL kids $(163.0 \pm 26.41$ and $130.9 \pm 26.41 \mathrm{nmol} / \mathrm{L}$, respectively) had similar cortisol concentrations to CAUT kids. We found no evidence that plasma glucose and lactate concentrations or body temperature were affected by treatment. The administration of isoflurane, with or without meloxicam, appeared to reduce pain associated with cautery disbudding.

Key words: goat kid, disbudding, physiology, pain relief, welfare

\section{INTRODUCTION}

In commercial housing systems, horned goats risk injuring themselves and conspecifics during agonistic interactions (Patt et al., 2012) and stockmen during handling (Smith and Sherman, 2009c). Furthermore, horned goats require more space in lying areas and at feed barriers (Loretz et al., 2004). Therefore, goat kids are routinely cautery disbudded within the first week of life (Smith and Sherman, 2009b). Disbudding causes burning and necrosis of tissue (increasing the risk of bacterial infection; Thompson et al., 2005), inflammation, and subcutaneous damage (Wright et al., 1983), all of which can cause pain. However, the use of pain relief is uncommon, and few studies (Alvarez et al., 2009; Ingvast-Larsson et al., 2011) have investigated its use during disbudding of kids.

Disbudding is associated with changes in behavior and physiology indicative of pain and distress. Behavioral responses during disbudding include frequent high-intensity vocalizations and struggling (Alvarez et al., 2009). Postdisbudding behavioral changes include increased head shaking, head scratching, head rubbing, self-grooming, and body shaking (Hempstead et al., 
2017) as well as isolation from penmates and increases in time spent motionless (Greenwood and Shutt, 1990; Ingvast-Larsson et al., 2011); therefore, the pain response may be characterized by vigorous head-oriented movements punctuated by motionless periods. Respiration rate, heart rate, and plasma cortisol, glucose, and $\beta$-endorphin concentrations have also been investigated in response to disbudding (Greenwood and Shutt, 1990; Ingvast-Larsson et al., 2011; Alvarez et al., 2015). Cortisol and $\beta$-endorphin concentrations were elevated in disbudded kids compared with controls (Greenwood and Shutt, 1990; Alvarez et al., 2015). With such evidence, it is necessary to evaluate pain relief strategies to reduce or eliminate pain associated with the practice.

Local anesthesia, such as lidocaine, can markedly reduce behavioral and physiological responses caused by disbudding in calves (Morisse et al., 1995; Graf and Senn, 1999); however, lidocaine does not consistently reduce pain-related behaviors (Chandrahas et al., 2013) or plasma cortisol concentrations (Alvarez et al., 2015; Nfor et al., 2016) in disbudded kids. An effective block using lidocaine may be difficult to achieve in kids, as the horns are supplied by the cornual branches of lacrimal and infratrochlear nerves, whereas calves only have the cornual branch of the lacrimal nerve innervating each horn bud (Dugdale, 2011). Additionally, young kids are sensitive to lidocaine and have an increased risk of toxicity at high doses (Smith and Sherman, 2009a). For these reasons, lidocaine was not investigated in the present study.

General anesthesia induced before disbudding may prevent acute pain. Isoflurane gas, a general anesthetic, can be used to safely anesthetize goats (McEwen et al., 2000; Dzikiti et al., 2011), but its use during disbudding of kids has not been evaluated. Analgesics, such as meloxicam (a nonsteroidal anti-inflammatory drug; NSAID), administered to kids after cautery disbudding resulted in fewer signs of pain (using a visual ana$\log$ scale from $1-10$, where $1=$ no signs and $10=$ severe signs of pain) than those disbudded without meloxicam (Ingvast-Larsson et al., 2011). These drugs are advantageous for on-farm use, as they can be administered orally or injected by farm staff under veterinarian supervision. However, NSAID reduce pain associated with inflammation, not acute pain caused by the destruction of nociceptors during disbudding (IngvastLarsson et al., 2011). The objective of our study was to evaluate isoflurane, either alone or in combination with meloxicam, and meloxicam administered orally or s.c. on goat kid behavior and physiology following cautery disbudding. We hypothesized that the behavioral and physiological responses to disbudding would be lower in kids administered isoflurane and meloxicam due to induced unconsciousness and mediation of inflamma- tory pain. We also hypothesized that kids administered s.c. meloxicam would display a reduced behavioral and physiological response to disbudding than kids administered oral meloxicam due to faster absorption rates (Karademir et al., 2016).

\section{MATERIALS AND METHODS}

This study was composed of 2 trials; trial 1 measured behavioral responses and trial 2 measured physiological responses. The trials were run separately to avoid having the physiological sampling protocols (e.g., blood sampling) affect the behavioral responses of animals.

\section{Animals and Location}

Trials were conducted in July and August (southern hemisphere winter) of 2014 (behavior) and 2015 (physiology) at the Ruakura Research Farm, Waikato, New Zealand $\left(37^{\circ} 47^{\prime} \mathrm{S}, 175^{\circ} 19^{\prime} \mathrm{E}\right)$, and approved by the Ruakura Animal Ethics Committee (Protocol No 13255). At 1 to $2 \mathrm{~d}$ of age, female Saanen cross dairy goat kids were collected from commercial farms, having been separated from their dams within $24 \mathrm{~h}$ of birth. Female kids were selected based on the following criteria: horn buds present (i.e., not polled), 2 to $7 \mathrm{~d}$ of age, of good health, and weighing $>2.5 \mathrm{~kg}$. On arrival at the Research Farm, kids were weighed and given an identification number. Stock-marking paint (Donaghys Sprayline, Christchurch, New Zealand) was used across the back of some animals (in a line or cross pattern), whereas others were left unmarked to enable individual identification during video analysis. Kids were fed approximately $300 \mathrm{~mL}$ of Anlamb milk replacer (Fonterra Ltd., Auckland, New Zealand) via a lamb feeding bucket, twice daily (at 0800 and $1600 \mathrm{~h}$ ). The feeders remained within the pens after feeding time with ad libitum access to further milk replacer.

\section{Study Facility}

The study facility had walls on all 4 sides and corrugated iron roofing. One large room contained 12 equalsized stalls $(3.60 \times 3.40 \times 1.15 \mathrm{~m}$ high $)$. The layout for trial 1 included 4 stalls, which comprised 6 smaller pens $(1.20 \times 1.64 \times 0.62 \mathrm{~m}$ high $)$ that housed animals pre- and post-treatment. Six additional stalls held the animals after a 2-d observation period ended. Another stall was allocated for kids that suffered from illness, and a final stall acted as the location for treatment administration. The pens that housed the animals for behavioral measurements were reconfigured for trial 2 . Five equal-sized stalls $(3.60 \times 3.40 \times 1.15 \mathrm{~m}$ high $)$ comprised 2 smaller pens $(1.64 \times 2.40 \times 0.62 \mathrm{~m}$ high $)$ : 
1 pen housed animals pretreatment and 1 housed the animals post-treatment. The other stalls remained the same as described for trial 1.

The pens were numbered and color coded to facilitate handling and identification of kids during video analysis. The concrete pen floors were covered with untreated wood shavings (Pinus radiata, PGG Wrightsons, Hamilton, New Zealand), $10 \mathrm{~cm}$ deep. Pens contained a plastic water trough $(15 \times 15 \times 17 \mathrm{~cm}$ deep $)$ fastened to the wall, and a milk feeder was placed on the dividing wall between adjacent pens.

\section{Experimental Design}

Our experiment used a randomized incomplete block design, using pen groups of 3 kids as the blocking variable. Treatments were balanced for age and the order of treatment was randomly generated by our biometrician using CycDesigN 5 (VSN International, Hemel Hempstead, UK). All kids that fulfilled the criteria described earlier were enrolled by the project manager (a research technician). At the time of enrolment, kids were allocated at random to 1 of 6 treatment groups (described below). The project manager was not blind to the treatments the kids received; however, handlers and other farm staff were blind to the treatment each kid received during both trials. Kids were fitted with a colored collar (blue, green, yellow, red, orange, or pink), associated with the treatment they received (handlers were blind to the treatment-color combinations), and were placed into their pretreatment pen groups (only 1 kid per treatment per pen as determined by the incomplete block randomization). The animals remained with the same penmates for the entire trial period. Both trials employed the same process to allocate treatments to kids.

A power analysis was carried out to determine the sample size required for both trials. The power analysis was based on a $5 \%$ significance level and with $80 \%$ power. The primary outcomes used to determine power size were struggling frequency and $\log 10$ blood cortisol, which were assumed to be normally distributed. The standard deviation and minimum response to be detected used were obtained from Alvarez et al. (2009) for struggling frequency (2.32 and 2.8, respectively) and from an ACTH challenge performed by our group (unpublished data) for cortisol (0.358 and 0.5, respectively).

On treatment days, kids were moved one at a time from the pretreatment pens into the treatment area and placed in a restraint device. This device was raised off the ground $(0.90 \mathrm{~m})$ and consisted of a rigid plastic pipe $(0.35 \times 0.85 \mathrm{~m})$ sectioned lengthways with holes for the kids' legs and straps to secure the kid across the shoulder and back. Hair covering the horn buds was removed with electric hair clippers (Laube, 505 cordless kit, Shoof, Cambridge, New Zealand) to expose the horn buds and to reduce hair burning. The restraint prevented movement at the time of treatment, so only the behaviors pre- and postrestraint were recorded. Kids were fed before treatment. All treatments were performed by a veterinarian between 0800 and $1600 \mathrm{~h}$ for trial 1 and between 0800 and $1000 \mathrm{~h}$ for trial 2 .

The treatments were as follows.

(1) Cautery disbudded (CAUT) using a modified version of the procedure described by IngvastLarsson et al. (2011) and without pain relief. The cautery iron (Quality electric debudder, 230 V, 190 W; Lister GmbH, Lüdenscheid, Germany) was heated for at least 20 min before use (approximately $600^{\circ} \mathrm{C}$ ) and held on each horn bud for $\leq 6 \mathrm{~s}$. The use of downwards pressure (and a circular motion of the cautery iron) on the animal cut the tissue, and with additional downward pressure, and a flick, forcibly lifted off the ring of tissue containing horn bud cells.

(2) Isoflurane gas (ISO) administered at a rate of $4 \%$ via a face mask (in oxygen); delivery continued until the animal lost consciousness (determined by dilation of pupils and loss of palpebral reflex), at which point the isoflurane mixing rate was reduced to $2 \%$. Kids were then disbudded using the same procedure as for CAUT. Following disbudding, isoflurane was removed from the gas supply, allowing the inhalation of pure oxygen for several seconds. The oxygen was then turned off, the face mask removed, and the animal remained in the restraint under supervision until it regained consciousness ( $\leq 5 \mathrm{~min})$.

(3) Isoflurane gas administered as in treatment 2 and meloxicam s.c. (ISO+MEL) administered using the same procedure described for I-MEL below, followed by cautery disbudding.

(4) Meloxicam (Loxicom 20mg $/ \mathrm{mL}$ solution for injection for cattle, pigs and horses, Norbrook Laboratories Ltd., Newry, UK), injected s.c. (IMEL; $0.5 \mathrm{mg} / \mathrm{kg}$ of BW) over the ribs, (dorsal thorax, caudal to the scapula), after which kids were disbudded using the same procedure as for CAUT.

(5) Meloxicam (Loxicom $0.5 \mathrm{mg} / \mathrm{mL}$ oral suspension for dogs, Norbrook Laboratories Ltd.) administered orally (O-MEL; $0.2 \mathrm{mg} / \mathrm{kg}$ of BW), after which kids were disbudded using the same procedure as for CAUT.

(6) Sham-handled (SHAM; simulated disbudding) with a cold disbudding iron applied to the horn 
buds (i.e., not disbudded) for $\leq 6 \mathrm{~s}$ and no pain relief given.

After horn bud removal, antibacterial spray (Tetravet, Bayer New Zealand Ltd., Auckland, New Zealand) was applied to the open wounds to prevent infection (this was not done on SHAM kids). The kids were then moved into the post-treatment pens.

Trial 1: Behavioral Responses. Seventy-two female Saanen cross dairy goat kids $(n=12 /$ treatment $)$ were used in this trial, consisting of 6 treatment days over $30 \mathrm{~d}$ with at least 6 kids treated per experimental day. Kids were aged between 2 and $7 \mathrm{~d}$ (mean \pm SEM; $3.9 \pm 0.15 \mathrm{~d}$ ) with BW ranging between 2.6 and $5.1 \mathrm{~kg}$ $(3.8 \pm 0.07 \mathrm{~kg})$.

Behaviors were continuously recorded using video cameras (HDR-CX220E, Sony Corp., Shanghai, China) for $24 \mathrm{~h}$ pre- and post-treatment. Due to the New Zealand industry standard age for disbudding kids, no time was allocated for habituation to the new environment before behavioral measurements commenced. The cameras were positioned at a height of $1.85 \mathrm{~m}$ above the pens (so that 1 camera could monitor 3 adjacent pens) and set at an angle of $30^{\circ}$ to the vertical attachment pole. Each camera was fitted with a fisheye lens (Raynox, Insta-Wide lenses, QC-303, Yoshida Industry Co. Ltd., Tokyo, Japan) to enable the whole width of each pen to be viewed. Red flood lights $\left(80 \mathrm{~W}, 30^{\circ}\right.$ beam angle, Philips Ltd., Auckland, New Zealand) were placed $2 \mathrm{~m}$ above each stall to assist with night observations.

Behaviors recorded were based on the ethogram described by Hempstead et al. (2017), but were modified to improve interobserver reliability (see Appendix Table A1 for ethogram comparison). Feeding was included in the present study as changes in feeding behavior have been reported in similar calf studies (Graf and Senn, 1999). Body shaking was consistently reliable between observers and therefore was not modified. The frequency of all behaviors was measured with the addition of duration of head scratching, self-grooming and feeding as these behaviors could be reliably measured.

One trained observer analyzed all video recordings using Adobe Premier Pro software (CS6, Version 6.0.0, Adobe Systems, San Jose, CA). When analyzing recordings, the observer remained blind to the treatment each kid received, except that SHAM kids could be identified as they were not disbudded or sprayed with blue antibiotic spray. Frequency and duration data were recorded against kid identification, which corresponded with the markings on the kids' backs (previously described) in the video recordings. Intraobserver reliability was performed on 3 kids over the same 1-h period, selected randomly from active periods, for each behavior $(\kappa=$
0.77 for head shaking; $\kappa=0.88$ for head scratching; $\kappa=0.98$ for self-grooming; $\kappa=0.95$ for feeding; and $\kappa=0.94$ for body shaking). Interobserver reliability between 3 observers was performed throughout observations by selecting a further 3 animals at random for each behavior (mean kappa among 3 observers, $\kappa=$ 0.88 for head shaking; $\kappa=1.00$ for head scratching; $\kappa$ $=0.79$ for self-grooming; $\kappa=0.93$ for feeding; and $\kappa=$ 1.00 for body shaking).

Lying behavior was measured using accelerometers (64k Pendant G data loggers, Onset Computer Corporation, Bourne, MA) set to record the $\mathrm{x}$ and $\mathrm{z}$ axes at $60-\mathrm{s}$ intervals. Lying duration was recorded continuously for $24 \mathrm{~h}$ pre- and post-treatment. The logger was placed into a durable fabric pouch and strapped (with hook and loop fasteners) to the lateral side of the left hind leg above the metatarsophalangeal joint. After the trial, data were downloaded using Onset HOBOware Pro software (Onset Computer Corporation, version 3.4.1) and converted to hourly summaries of lying behavior using SAS software (version 9.4, SAS Institute Inc., Cary, NC) code designed for this purpose; based on the work of Zobel et al. (2015), we corrected for single standing and lying events.

Trial 2: Physiological Responses. Sixty female Saanen cross dairy goat kids $(\mathrm{n}=10 /$ treatment $)$ were used; the trial included 4 treatment days over an 8-d period with a minimum of 12 kids treated per experimental day. Kids were aged between 3 and $6 \mathrm{~d}$ (mean \pm SEM; $4.3 \pm 0.14 \mathrm{~d}$ ) with BW ranging between 2.3 and $4.4 \mathrm{~kg}(3.3 \pm 0.06 \mathrm{~kg})$. Kids were given $24 \mathrm{~h}$ to habituate to their home pen before the trial commenced; they were therefore older than trial 1 animals.

Plasma cortisol, glucose, and lactate concentrations were measured using blood samples $(4 \mathrm{~mL})$ collected by venipuncture from the jugular veins immediately pretreatment (baseline; before hair clipping) and at 15, 60, and 120 min post-treatment. Two handlers, trained by a veterinarian, collected the blood samples from each kid; the process took $\leq 2.5 \mathrm{~min} / \mathrm{kid}$. Each kid was firmly restrained by a handler while samples were collected using 21-gauge 1-inch needles (PrecisionGlide, Becton Dickinson Vacutainer Systems, Franklin Lakes, NJ); samples were then held in fluoride oxalate tubes (Becton Dickinson Vacutainer Systems) on ice. Blood samples were centrifuged at 3,000 rpm (approximately $1,500 \times g$ ) for $10 \mathrm{~min}$ at $4^{\circ} \mathrm{C}$ immediately after sampling and the plasma was separated and stored at $-20^{\circ} \mathrm{C}$ until analyzed. Plasma cortisol, glucose, and lactate concentrations were analyzed in an independent laboratory by technicians blind to the treatments. Plasma concentrations of cortisol were determined by electrochemiluminescence immunoassay using a commercial kit (Roche Diagnostics GmbH, Mannheim, Germany). Sensitivity 
of the assay was $1.5 \mathrm{nmol} / \mathrm{L}$, and intra- and interassay coefficients of variation were 2.1 and $5.2 \%$, respectively. The plasma glucose concentration was determined by the hexokinase method using a commercial kit (Roche Diagnostics $\mathrm{GmbH}$ ) and the sensitivity of the assay was $0.1 \mathrm{mmol} / \mathrm{L}$. The plasma lactate concentration was determined by enzymatic methods using a commercial kit (Roche Diagnostics $\mathrm{GmbH}$ ) and the sensitivity of the assay was $0.2 \mathrm{mmol} / \mathrm{L}$. Body temperature was measured immediately after blood sampling at all 4 time points using a rectal Rapid Digital Thermometer (Vet Temp, Advanced Monitors Corp., San Diego, CA).

\section{Statistical Analysis}

Data were analyzed using Genstat software (Version 17, VSN International, Hemel Hempstead, UK). We inspected the residual diagnostic plots to detect departures from the assumptions of normality and constant variance. Log transformations were required for all behavior data except for head shaking and lying. Transformations of the physiological data were not required. We recorded no adverse events for trial 1, hence no kids were excluded. One animal was removed from trial 2 due to illness and another was excluded from the cortisol analysis due to a processing error. Total frequencies and durations of behaviors were grouped into 1-h periods across the 48-h observation period (pre- and post-treatment). In addition, frequency and duration data were grouped into twelve 5-min periods $1 \mathrm{~h}$ pre- and post-treatment to assess the acute behavioral response following treatment. Acute behavioral responses within $1 \mathrm{~h}$ post-treatment (during daylight hours) were chosen, as kids were most active within this period and plasma cortisol concentrations of goat kids typically return to baseline levels $1 \mathrm{~h}$ after disbudding (Alvarez et al., 2015).

Head shaking, head scratching, self-grooming, feeding, body shaking, body temperature, and plasma cortisol, glucose, and lactate concentrations from pre- to post-treatment were assessed using a repeated measures model fitted by REML. The model included the fixed effects of treatment, time, and their interaction and the random effects of kid identification, age, weight, farm, treatment date, and pen. The correlation between measurements taken on the same kid over time was modeled with a power model of order 1 . Further analyses were carried out on behavior data within the first hour post-treatment (twelve 5-min periods) using a repeated measures model fitted by REML. The model used the same variables as for the hourly analysis, except, in this case, the average of the twelve 5 -min periods $1 \mathrm{~h}$ pretreatment was used as a covariate.
Lying behavior was modeled using a repeated measures model fitted by REML, with the average of the pretreatment lying duration per hour as a covariate. The fixed and random variables were the same as above. To meet the assumptions of constant variance and normality, the lying data required rank transformation.

Differences between and within treatments were detected using Fisher's least significant differences test. Mean values (back-transformed if required, with exact $95 \% \mathrm{CI}$ ) were provided with standard errors of the difference. The level of significance was set at $P \leq 0.05$.

\section{RESULTS}

\section{Trial 1: Behavioral Responses}

We found no treatment by time interaction on the mean number of head shakes per hour over the 48-h observation period $(P=1.00)$; however, we noted an overall effect of time $\left(F_{48,3033}=18.3 ; P<0.001\right)$. Head shakes were 10 times more frequent $1 \mathrm{~h}$ posttreatment compared with pretreatment and all other post-treatment hours $(P \leq 0.05)$. For the 5 -min periods within $1 \mathrm{~h}$ post-treatment, we observed a treatment by time interaction $\left(F_{55,672}=1.6 ; P=0.005\right)$. At 5 min post-treatment, CAUT kids performed over double the number of head shakes as SHAM kids $(P \leq 0.05)$ but displayed similar levels as SHAM kids by $10 \mathrm{~min}$ post-treatment $(P=0.93)$. In addition, ISO+MEL kids performed half as many head shakes as CAUT kids 5 min post-treatment and ISO kids performed one-third the number of head shakes as CAUT kids for up to 10 min post-treatment $(P \leq 0.05)$. Both ISO+MEL and ISO kids performed less head shaking than I-MEL and O-MEL kids at 5 min post-treatment $(P \leq 0.05)$. Both I-MEL and O-MEL kids performed similar frequencies of head shaking to CAUT and SHAM kids over the twelve 5-min periods within $1 \mathrm{~h}$ post-treatment $(P>$ 0.08) except for I-MEL kids, which performed twice as many head shakes as SHAM kids at 15 min posttreatment $(P=0.05)$.

We found no treatment by time interaction on the mean number of body shakes per hour over $48 \mathrm{~h}$ (preand post-treatment; $P=1.00)$; however, we did note an effect of time $\left(F_{48,3030}=16.3 ; P<0.001\right)$. Overall, kids had a 3 -fold increase in body shakes $1 \mathrm{~h}$ post-treatment compared with pretreatment hours $(P \leq 0.05)$. There was a treatment by time interaction across the 5 -min periods $1 \mathrm{~h}$ post-treatment for body shaking frequencies $\left(F_{55,672}=2.1 ; P<0.001\right)$. At 5 min post-treatment, CAUT kids performed, on average, 3 times as many body shakes as SHAM kids $(P \leq 0.05)$, but declined 
to SHAM kid frequencies during the remainder of the hour $(P>0.15)$. Furthermore, ISO+MEL and ISO kids performed less than one-quarter the number of body shakes as CAUT kids 5 min post-treatment $(P \leq 0.05)$. At 10 min post-treatment, ISO kids performed onethird the number of body shakes as CAUT kids $(P \leq$ $0.05)$. Additionally, ISO+MEL and ISO kids performed one-fifth the number of body shakes as I-MEL and OMEL kids at 5 min post-treatment $(P \leq 0.05)$.

We observed a treatment by time interaction for hourly head scratching durations over $48 \mathrm{~h}\left(F_{235,2952}=\right.$ 1.4; $P<0.001$; Figure 1). At $1 \mathrm{~h}$ post-treatment, CAUT kids spent more time head scratching than SHAM kids $(P \leq 0.05)$. In addition, I-MEL and ISO+MEL had higher head scratching durations than SHAM kids $(P \leq 0.05)$, and O-MEL kids spent more time head scratching than SHAM kids but reduced to SHAM kid levels at $2 \mathrm{~h}$ post-treatment $(P>0.24$; Figure 1$)$. Over the post-treatment period, ISO and ISO+MEL kids performed similar head scratching durations as CAUT kids $(P>0.18)$; however, ISO+MEL kid durations were elevated above CAUT kid levels $5 \mathrm{~h}$ post-treatment $(P=0.001$; Figure 1). For O-MEL kids, time spent head scratching was less than for I-MEL kids at many time points post-treatment $(P \leq 0.05$; Figure 1$)$. There was no treatment by time interaction on mean head scratching frequencies over $48 \mathrm{~h}(P=0.27)$, although we did note an overall effect of time $\left(F_{48,3034}=9.9\right.$; $P<0.001)$. All kids performed twice as many head scratches $1 \mathrm{~h}$ post-treatment than $1 \mathrm{~h}$ pretreatment $(P$ $\leq 0.05$ ). We found no difference between treatments within $1 \mathrm{~h}$ post-treatment for head scratching frequencies across the twelve 5 -min periods $(P=0.44)$.

We noted no treatment or treatment by time interaction on self-grooming frequencies and durations over 48 $\mathrm{h}(P>0.92)$. There was an effect of time over $48 \mathrm{~h}$ on self-grooming, with a 2 -fold increase in the activity over $12 \mathrm{~h}$ post-treatment compared with the pretreatment period $\left(F_{48,3035}=11.6 ; P<0.001\right)$.

No effect of treatment or treatment by time interaction was observed on feeding frequency and duration over $48 \mathrm{~h}(P=1.00)$, but we noted a separate effect of time on feeding $\left(F_{48,3034}=7.33 ; P<0.001\right)$. Kids fed more often $2 \mathrm{~h}$ pretreatment compared with $1 \mathrm{~h}$ posttreatment $(P \leq 0.05)$. Furthermore, feeding frequency was higher $4 \mathrm{~h}$ post-treatment compared with $1 \mathrm{~h}$ posttreatment $(P \leq 0.05)$. Kids spent more time feeding during the first half of the post-treatment period compared with the pretreatment period $(P \leq 0.05)$.

Lying duration was similar across treatments over $24 \mathrm{~h}$ post-treatment $(P=1.00)$, although we found an effect of time on lying behavior $\left(F_{23,606}=14.6 ; P\right.$ $<0.001)$. For all treatments, kids spent more time on average lying (3.5 times) $2 \mathrm{~h}$ post-treatment than $1 \mathrm{~h}$ post-treatment $(P \leq 0.05)$.

\section{Trial 2: Physiological Responses}

We observed no treatment by time interaction for cortisol concentrations $(P=0.24)$; however, there were separate effects of time $\left(F_{3,118}=58.8 ; P<0.001\right)$ and treatment $\left(F_{5,43}=2.5 ; P=0.04\right.$; Figure 2$)$. Baseline cortisol concentrations were similar across treatments $\left(125.5 \pm 11.81 \mathrm{nmol} / \mathrm{L} ; F_{5}=1.1 ; P=0.37\right)$. Overall, cortisol concentrations peaked at 15 min post-treatment and dropped below baseline at $60 \mathrm{~min}$ post-treatment $(P \leq 0.05)$. Cortisol concentrations for CAUT kids were higher than SHAM kids over the 2-h post-treatment sampling period $(P \leq 0.05)$. Moreover, cortisol concentrations were similar between ISO+MEL and SHAM kids $(P=0.47)$ but lower than CAUT kids $(P \leq 0.05)$; O-MEL kids had similar cortisol concentrations to CAUT kids $(P=0.86)$. Furthermore, ISO and I-MEL kids had similar cortisol concentrations to both CAUT and SHAM kids $(P>0.24)$.

We found no treatment by time interaction for glucose concentrations $(P=0.68$; Table 1$)$ or an effect of treatment $(P=0.12)$; however, a separate effect of time was noted $\left(F_{3,162}=5.3 ; P=0.002\right)$. Glucose concentrations were higher $(P \leq 0.05) 2 \mathrm{~h}$ post-treatment than at baseline. There was no treatment by time interaction for lactate concentrations $(P=0.36$; Table 1$)$ or separate effects of time $(P=0.30)$ and treatment $(P=$ 0.45 ). We observed no treatment by time interaction for body temperature $(P=0.28$; Table 1$)$ or an effect of treatment $(P=0.70)$; however, we did note a separate effect of time $\left(F_{3,116}=3.5 ; P=0.02\right)$. Body temperature was higher for all kids at 15 min post-treatment than baseline $(P \leq 0.05)$.

\section{DISCUSSION}

Our study is the first to examine the effects of inducing general anesthesia using isoflurane, either alone or in combination with an NSAID (meloxicam), on the behavioral and physiological responses of goat kids following cautery disbudding. General anesthetic (alone or in combination with an oral NSAID) reduced the behavioral response of kids immediately after disbudding, particularly head and body shakes; therefore, our hypothesis, that unconsciousness reduces subsequent responsiveness, was supported. However, it is possible that behavior was affected by the isoflurane alone. An additional sham-handled group provided anesthesia but not disbudded may have displayed a similar response to the ISO group; however, this treatment was not in- 
A

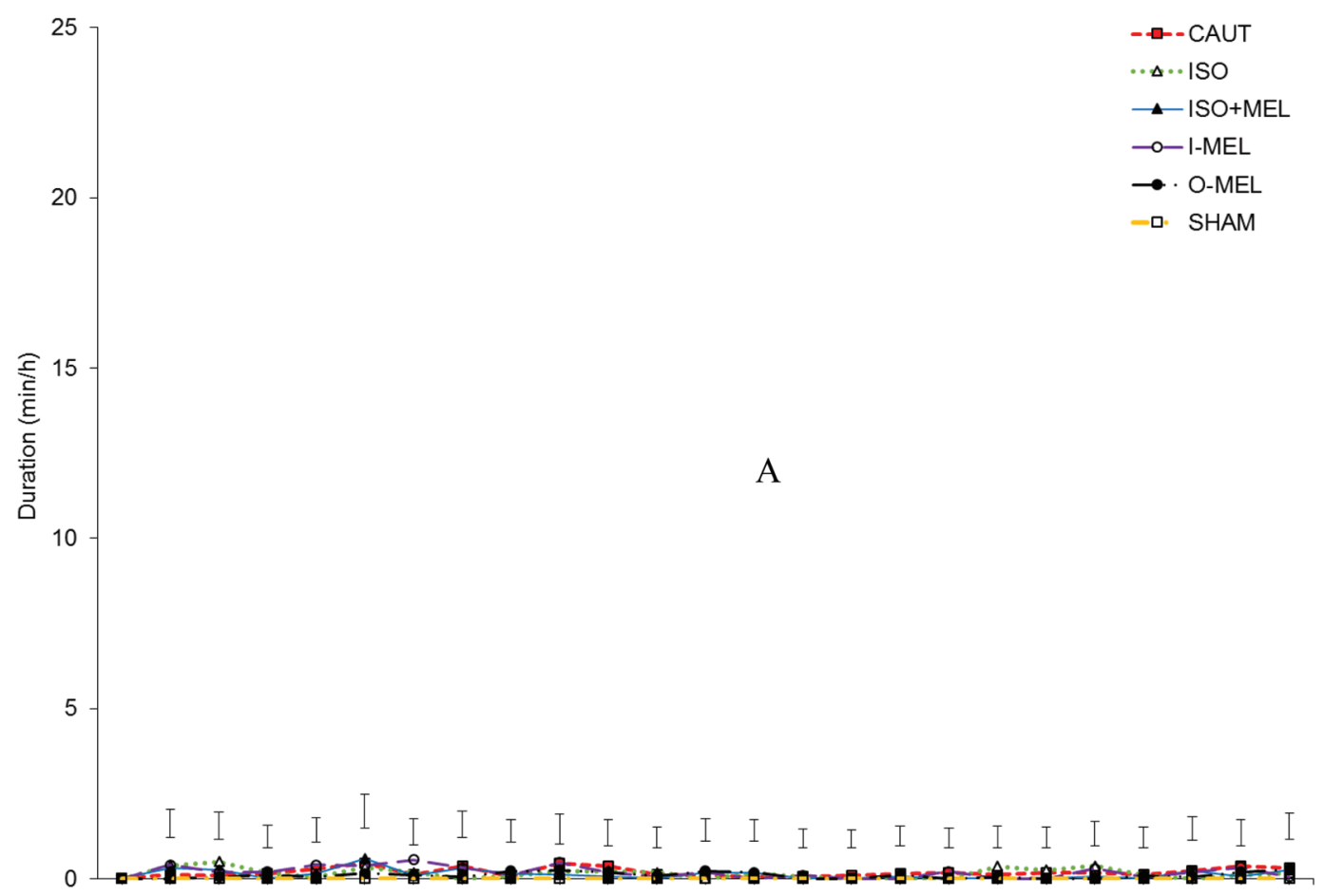

B

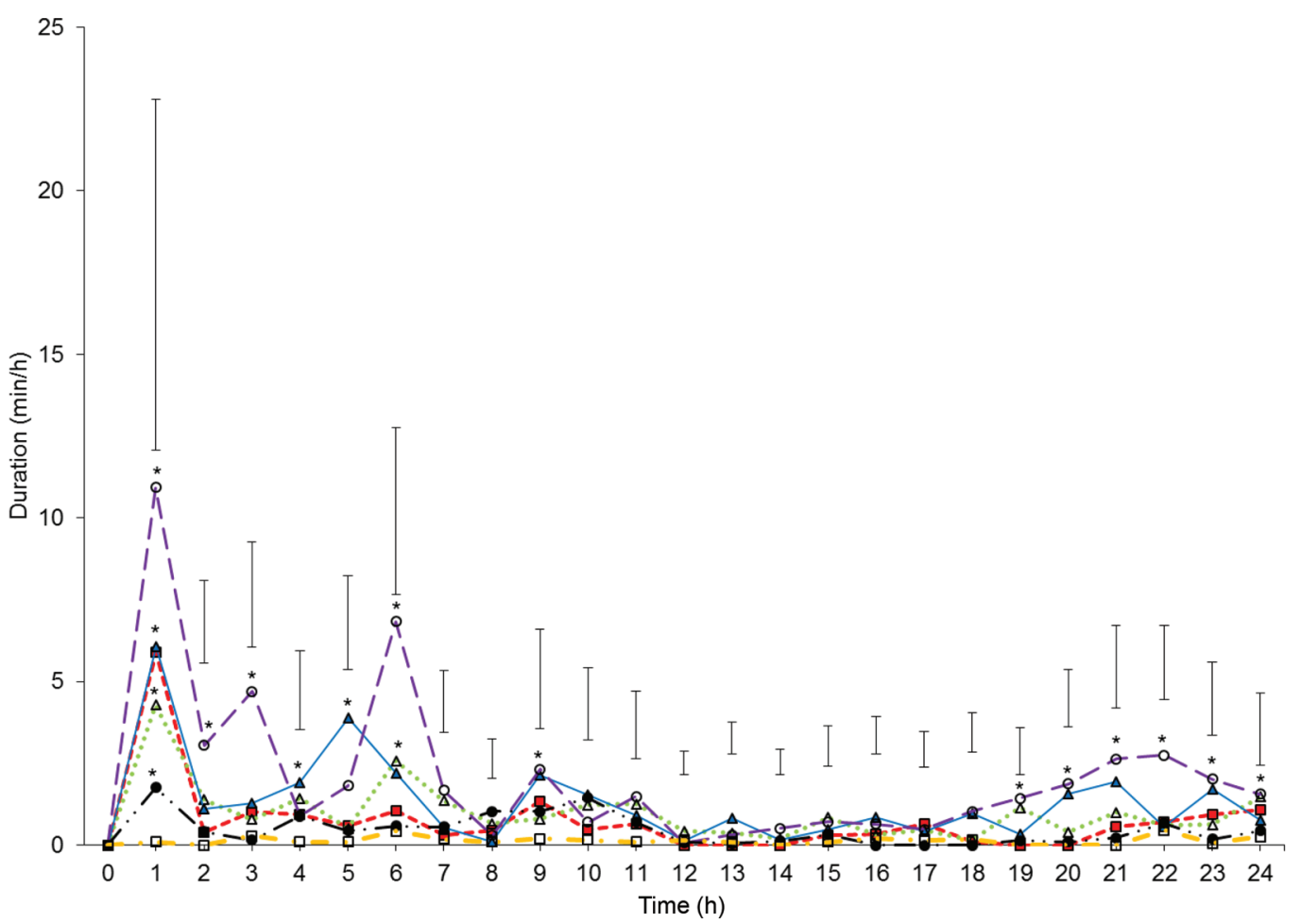

Figure 1. Back-transformed mean head scratching durations (min/h; error bars represent exact $95 \% \mathrm{CI}$ ) over $24 \mathrm{~h}$ pre-treatment (A) and $24 \mathrm{~h}$ post-treatment $(\mathrm{B})$ of goat kids exposed to experimental treatments $(\mathrm{n}=12 /$ treatment). An asterisk $(*)$ indicates means that differ from SHAM kid means at $P<0.05$. CAUT $=$ disbudded with no pain relief; ISO = disbudded with isoflurane gas; ISO+MEL $=$ disbudded with isoflurane and s.c. meloxicam; I-MEL = disbudded with meloxicam s.c. (0.5 mg/kg of BW); O-MEL = disbudded with oral meloxicam (0.2 mg/ $\mathrm{kg}$ of BW); SHAM = sham-handled only (simulated disbudding). Color version available online. 


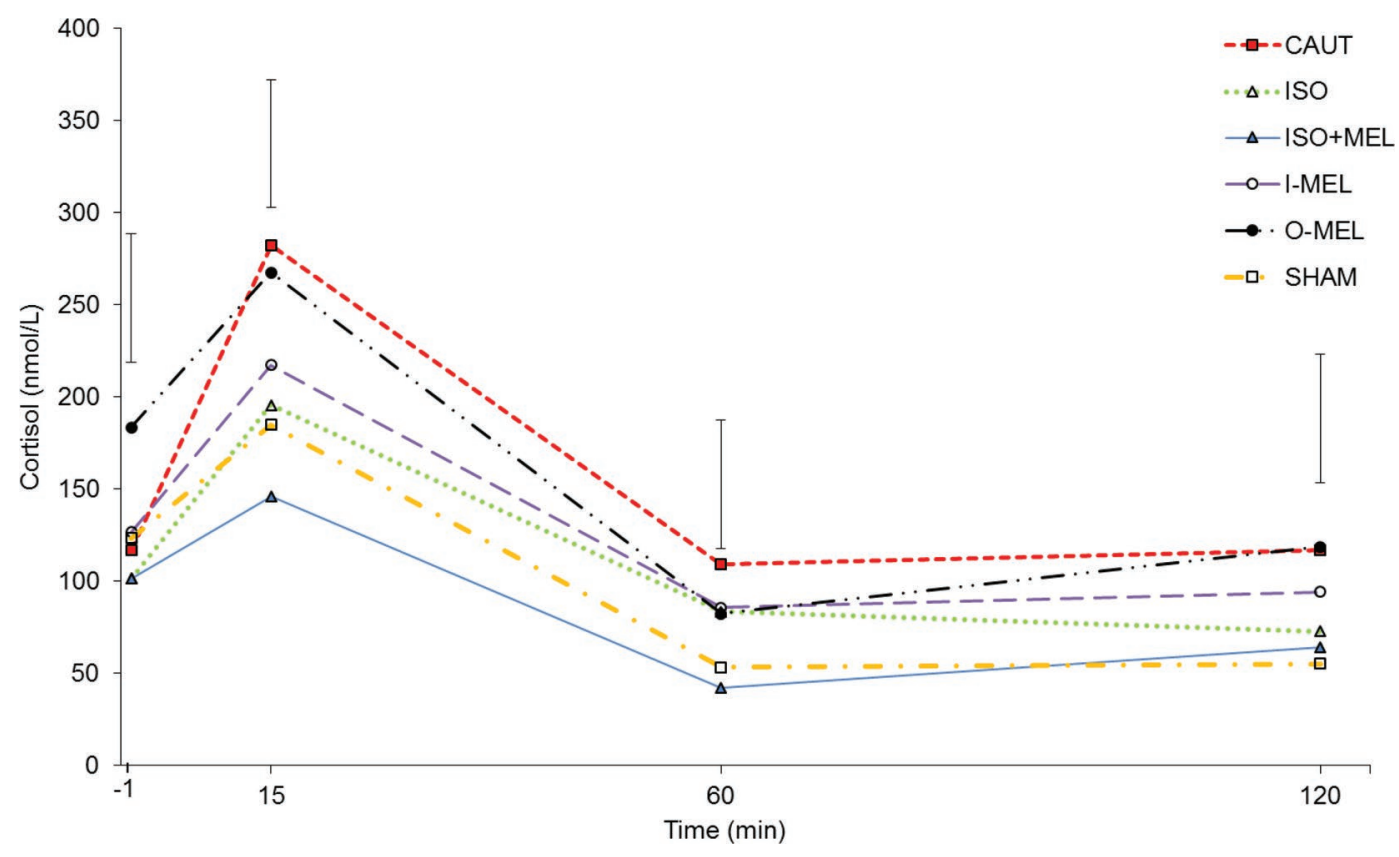

Figure 2. Plasma cortisol concentrations (nmol/L; mean \pm standard error of the difference) over $2 \mathrm{~h}$ post-treatment of goat kids exposed to experimental treatments $(\mathrm{n}=12 /$ treatment $)$. CAUT $=$ disbudded with no pain relief; ISO $=$ disbudded with isoflurane gas; ISO $+\mathrm{MEL}=$ disbudded with isoflurane and s.c. meloxicam; I-MEL = disbudded with meloxicam s.c. $(0.5 \mathrm{mg} / \mathrm{kg}$ of BW $)$; O-MEL = disbudded with oral meloxicam $(0.2 \mathrm{mg} / \mathrm{kg}$ of BW); SHAM = sham-handled only (simulated disbudding). Color version available online.

Table 1. Plasma glucose [mmol/L; mean and standard error of the difference (SED)] and lactate concentrations (mmol/L; mean and SED) and body temperatures $\left({ }^{\circ} \mathrm{C}\right.$; mean and SED) of goat kids over $2 \mathrm{~h}$, from 1 min pretreatment to 120 min post-treatment $(\mathrm{n}=12 /$ treatment $)$

\begin{tabular}{|c|c|c|c|c|}
\hline \multirow[b]{2}{*}{ Item $^{1}$} & \multicolumn{4}{|c|}{ Time $(\min )$} \\
\hline & -1 & 15 & 60 & 120 \\
\hline \multicolumn{5}{|c|}{ Glucose $(\mathrm{mmol} / \mathrm{L})$} \\
\hline CAUT & 6.3 & 6.7 & 7.1 & 7.9 \\
\hline ISO & 6.1 & 6.0 & 6.4 & 6.8 \\
\hline ISO+MEL & 6.6 & 6.4 & 6.7 & 7.4 \\
\hline I-MEL & 5.2 & 5.7 & 6.1 & 6.4 \\
\hline O-MEL & 5.7 & 6.1 & 6.1 & 5.9 \\
\hline SHAM & 5.7 & 6.2 & 6.3 & 6.4 \\
\hline Average SED & 0.70 & 0.62 & 0.66 & 0.68 \\
\hline \multicolumn{5}{|c|}{ Lactate $(\mathrm{mmol} / \mathrm{L})$} \\
\hline CAUT & 2.4 & 2.7 & 2.2 & 2.3 \\
\hline ISO & 2.3 & 2.3 & 2.5 & 2.4 \\
\hline ISO+MEL & 2.3 & 2.8 & 2.9 & 2.6 \\
\hline I-MEL & 1.9 & 2.5 & 2.4 & 2.4 \\
\hline O-MEL & 2.9 & 2.6 & 2.5 & 2.5 \\
\hline SHAM & 2.2 & 2.6 & 2.4 & 3.0 \\
\hline Average SED & 0.42 & 0.39 & 0.41 & 0.41 \\
\hline \multicolumn{5}{|c|}{ Body temperature $\left({ }^{\circ} \mathrm{C}\right)$} \\
\hline CAUT & 38.6 & 39.2 & 39.0 & 38.9 \\
\hline ISO & 38.8 & 38.9 & 38.8 & 38.9 \\
\hline ISO+MEL & 39.1 & 39.1 & 39.2 & 39.2 \\
\hline I-MEL & 38.8 & 39.0 & 38.9 & 38.8 \\
\hline O-MEL & 39.0 & 39.3 & 38.9 & 38.8 \\
\hline SHAM & 38.9 & 39.0 & 38.9 & 39.1 \\
\hline Average SED & 0.25 & 0.23 & 0.23 & 0.23 \\
\hline
\end{tabular}


cluded in the present study due to logistical reasons. Isoflurane may have affected kid behavior for up to 2 $\mathrm{h}$ post-treatment, as has been observed in calves that had undergone umbilical surgery (Offinger et al., 2012). Therefore, future research is required to establish the longer-term effects of isoflurane on kid behavior.

The behavioral response of disbudded kids appears relatively short-lived in comparison with disbudded calves, which exhibit higher frequencies of head shaking than sham controls for up to $4 \mathrm{~h}$ after disbudding (Graf and Senn, 1999; Grondahl-Nielsen et al., 1999; Heinrich et al., 2010). In the present study, the behavioral response diminished $1 \mathrm{~h}$ after treatment. A potential explanation is the difference in age that disbudding is typically performed, as kids are usually disbudded within a week of age (Smith and Sherman, 2009b) and calves usually between 4 to 6 wk; therefore, the horn buds of calves are more developed than those of 1-wkold kids. Furthermore, the amount of time taken to disbud calves and kids can differ; in calves, disbudding can take up to $20 \mathrm{~s}$ per horn bud (Graf and Senn, 1999) compared with approximately $6 \mathrm{~s}$ per horn bud for kids in the present study. Thus, cautery disbudding in kids may be less painful than disbudding in calves.

In the present study, isoflurane, whether in combination with meloxicam or not, reduced the cortisol response to disbudding in kids for up to $2 \mathrm{~h}$, which indicates a reduction in acute pain associated with the practice. This is in line with research that found calves disbudded with local anesthesia with or without sedation showed a reduced cortisol response (GrondahlNielsen et al., 1999). Yet others reported that local anesthesia did not reduce the cortisol response of disbudded calves compared with calves not provided pain relief (Morisse et al., 1995). The difference in results may be associated with difficulty in administering a successful nerve block, which is also apparent in goat kids (Alvarez et al., 2009). Furthermore, in the present study, isoflurane (with or without meloxicam) is more effective than meloxicam alone, regardless of the route of administration. Interestingly, Heinrich et al. (2009) reported calves disbudded with meloxicam had lower serum cortisol than saline controls $6 \mathrm{~h}$ after disbudding, indicating that meloxicam reduced the physiological stress response to disbudding. As the response of goat kids to disbudding appears short in duration, perhaps the long-term action of meloxicam is not as apparent as it is for calves. Isoflurane, with or without meloxicam, shows promise as a method of pain relief for disbudding goat kids; however, the use of isoflurane anesthesia on farm may not be practical due to the time required for recovery and equipment and personnel requirements (e.g., veterinary administration), but a portable isoflu- rane delivery device could be developed for on-farm use. Meloxicam is better suited to on-farm use, as it can be administered by farm staff, but may be less effective.

We hypothesized that kids provided injected meloxicam would have a lower behavioral and physiological response to disbudding than kids provided oral meloxicam due to rapid absorption rates. However, oral meloxicam appeared to generate a lower behavioral response than injected meloxicam. It is unclear why oral meloxicam would be more effective than injected meloxicam at reducing pain, as the subcutaneous route should be absorbed into the blood quicker than oral meloxicam (Ingvast-Larsson et al., 2011; Karademir et al., 2016). Ingvast-Larsson et al. (2011) reported that disbudded goat kids administered injected meloxicam showed less signs of pain (using a visual analog score) than groups disbudded without meloxicam. Therefore, evidence suggests that oral meloxicam may reduce postoperative pain associated with cautery disbudding in goat kids. Plasma cortisol concentrations of kids receiving the NSAID were similar to cautery disbudded kids without pain relief, which suggests that meloxicam, regardless of the route of administration, may not reduce the acute stress response to disbudding. This is in line with earlier literature reporting that cortisol concentrations were similar between kids disbudded with and without NSAID (Ingvast-Larsson et al., 2011). In comparison with calf literature, meloxicam or ketoprofen administered $10 \mathrm{~min}$ before disbudding resulted in lower cortisol concentrations than calves without NSAID for up to $6 \mathrm{~h}$ (Milligan et al., 2004; Heinrich et al., 2009) and reduced head shaking from 3 to $24 \mathrm{~h}$ postdisbudding (Faulkner and Weary, 2000). In the present study, on average, kids given meloxicam along with isoflurane had a lower behavioral and physiological response to disbudding; therefore, used in combination with anesthesia, meloxicam may reduce pain. In the present study, both injected and oral meloxicam were given immediately before disbudding to model on-farm application (i.e., to avoid double handling of animals). Future research is required to establish the optimal time of meloxicam administration before disbudding in goat kids.

Our earlier findings suggested that head scratching and self-grooming frequencies may reflect pain associated with cautery disbudding (Hempstead et al., 2017). Yet in the present study, we found no statistically significant differences between treatments either (1) over $48 \mathrm{~h}$ pre- and post-treatment or (2) within $1 \mathrm{~h}$ post-treatment for head scratching and self-grooming frequencies. A key difference between these studies was the use of different ethograms, which were modified to increase interobserver reliability. However, the mean 
values for head scratching frequency in the present study tended to be higher in cautery disbudded than sham-handled controls.

No differences in feeding behavior were found across treatments, which is consistent with our earlier study (Hempstead et al., 2017); however, in calves, feeding behavior is affected for up to $4 \mathrm{~h}$ after disbudding (Graf and Senn, 1999). It appears that all kids, regardless of treatment, fed more often after handling or treatment than before, which may be associated with the lack of sufficient habituation before behavior recording commenced or that the kids had learned how to feed independently. In addition, lying behavior of kids over $24 \mathrm{~h}$ post-treatment did not differ significantly between treatments, which contrasts with Chandrahas et al. (2013), who reported that sleeping duration was higher in kids provided meloxicam than kids disbudded only. Differences in results could be due to differences in how lying or sitting behavior were measured; Chandrahas et al. (2013) recorded behavior manually by observation with a point sampling method, whereas we measured lying behavior continuously using accelerometers. It has also been observed in disbudded calves that lying behavior was similar between groups provided with pain relief or without (Faulkner and Weary, 2000; Duffield et al., 2010; Sutherland et al., 2016).

In the present study, basal cortisol concentrations were higher $1 \mathrm{~min}$ pre- than $1 \mathrm{~h}$ post-treatment for all kids, which may be related to the timing of feeding and associated handling of the kids approximately half an hour before disbudding. Adult goats have shown increased plasma cortisol within 60 min of feeding (Eriksson and Terāväinen, 1989). In dairy cows, cortisol is known to be released in response to feeding (Willett and Erb, 1972). In future studies, a longer settling period after feeding may be required to enable cortisol concentrations to return to basal levels before testing. Periodic fluctuations (e.g., circadian rhythms) in glucocorticoid levels, that have been previously described in other mammalian species such as pigs (Andersson et al., 2000) and horses (Irvine and Alexander, 1994), may explain the difference in cortisol over the blood sampling period; however, goats do not appear to show this daily rhythm in cortisol (Alila-Johansson et al., 2003).

Gluconeogenesis, which is the process of synthesizing glucose, is stimulated by cortisol and other glucocorticoids. Prunier et al. (2005) reported elevations in cortisol in castrated pigs but no increase in plasma glucose compared with uncastrated controls, which was most likely due to insufficient glycogen stores in young pigs. We found no statistically significant differences in mean glucose concentrations between treatments, which is consistent with previous goat studies (e.g., Ingvast-Larsson et al., 2011); furthermore, we found no statistically significant differences among treatments for mean lactate, which typically increases with stress or pain in pigs (Prunier et al., 2005) and dairy cattle (El-Ghoul and Hofmann, 2002). The difference in glucose and lactate elevation between disbudding and castration may be associated with castration causing more visceral pain than disbudding, which may cause more somatic pain (Dunckley et al., 2005) or high variability of blood constituents in goat kids due to early life development (Chen et al., 1999). In addition, we found no evidence that mean body temperature was affected by our treatments; however, overall kids had higher body temperature after treatment, indicating that handling and associated stress affected body temperature rather than the treatments themselves. This contrasts with the findings of Nfor et al. (2016), who reported that body temperature decreased postdisbudding compared with baseline values in kids that were disbudded either under sedation (dexmedetomidine hydrochloride) or provided a local ring block (1 mL s.c.). However, body temperature was not reduced in kids disbudded without pain relief (injected saline); therefore, differences were likely caused by the pain relief treatments.

\section{CONCLUSIONS}

Cautery disbudding causes elevated cortisol concentrations, increased head shaking and body shaking frequencies, and greater head scratching durations. Isoflurane alone or in combination with meloxicam reduced the behavioral and cortisol responses to cautery disbudding and therefore may significantly reduce pain. Furthermore, meloxicam appears to reduce postoperative pain, but more research on the optimal time to administer meloxicam before disbudding is needed. Although the use of isoflurane (with or without meloxicam) can reduce pain associated with cautery disbudding, the practicality of using isoflurane on farm needs to be addressed; other pain relief options should also be evaluated to improve the welfare of disbudded goat kids.

\section{ACKNOWLEDGMENTS}

We are grateful for assistance provided by AgResearch staff especially Trevor Watson, Frances Huddart, Stephany Delaney, Rose Greenfield, Ariane Bright, and Bridget Wise. We also thank Ali Cullum for her many contributions to this study and performing treatment administration. This work was supported by the New Zealand Ministry of Business, Innovation and Employment (MBIE: Contract C10X1307; Wellington, New Zealand) and the Dairy Goat Cooperative (DGC; Hamilton, New Zealand) Ltd. 


\section{REFERENCES}

Alila-Johansson, A., L. Eriksson, T. Soveri, and M.-L. Laakso. 2003. Serum cortisol levels in goats exhibit seasonal but not daily rhythmicity. Chronobiol. Int. 20:65-79.

Alvarez, L., J. B. De Luna, D. Gamboa, M. Reyes, A. Sánchez, A. Terrazas, S. Rojas, and F. Galindo. 2015. Cortisol and pain-related behavior in disbudded goat kids with and without cornual nerve block. Physiol. Behav. 138:58-61.

Alvarez, L., R. A. Nava, A. Ramirez, E. Ramirez, and J. Gutierrez. 2009. Physiological and behavioral alterations in disbudded goat kids with and without local anaesthesia. Appl. Anim. Behav. Sci. 117:190-196.

Andersson, H., K. Lillpers, L. Rydhmer, and M. Forsberg. 2000. Influence of light environment and photoperiod on plasma melatonin and cortisol profiles in young domestic boars, comparing two commercial melatonin assays. Domest. Anim. Endocrinol. 19:261-274.

Chandrahas, A. L. Saini, D. S. Malik, and J. Mohindroo. 2013. Effect of local anaesthetics and non-steroidal analgesics on maintenace behavior of hot-iron disbudded beetal kids under stall-fed conditions. Indian J. Anim. Res. 47:407-411.

Chen, J. C., C. J. Chang, H. C. Peh, and S. L. Lee. 1999. Perinatal adrenocortical function in relation to the growth rate and immunoglobulin acquisition of goat kids. Small Rumin. Res. 33:255-262.

Duffield, T. F., A. Heinrich, S. T. Millman, A. DeHaan, S. James, and K. Lissemore. 2010. Reduction in pain response by combined use of local lidocaine anesthesia and systemic ketoprofen in dairy calves dehorned by heat cauterization. Can. Vet. J. 51:283-288.

Dugdale, A. 2011. Veterinary Anaesthesia: Principles to Practice. Wiley, Hoboken, NJ.

Dunckley, P., R. G. Wise, M. Fairhurst, P. Hobden, Q. Aziz, L. Chang, and I. Tracey. 2005. A Comparison of visceral and somatic pain processing in the human brainstem using functional magnetic resonance imaging. J. Neurosci. 25:7333-7341.

Dzikiti, T. B., G. F. Stegmann, L. N. Dzikiti, and L. J. Hellebrekers. 2011. Effects of midazolam on isoflurane minimum alveolar concentration in goats. Small Rumin. Res. 97:104-109.

El-Ghoul, W., and W. Hofmann. 2002. Influence of claw diseases of varying degrees on measurable stress reactions with special consideration of cortisol and lactate in bovine blood serum. Prakt. Tierarzt 83:354-361.

Eriksson, L., and T. L. Terāväinen. 1989. Circadian rhythm of plasma cortisol and blood glucose in goats. Asian-Australas. J. Anim. Sci. 2:202-203.

Faulkner, P. M., and D. M. Weary. 2000. Reducing pain after dehorning in dairy calves. J. Dairy Sci. 83:2037-2041.

Graf, B., and M. Senn. 1999. Behavioral and physiological responses of calves to dehorning by heat cauterization with or without local anaesthesia. Appl. Anim. Behav. Sci. 62:153-171.

Greenwood, P. L., and D. A. Shutt. 1990. Effects of management practices on cortisol, b-endorphin and behavior in young goats. Pages 224-227 in Proc. Aust Soc Anim Prod., Adelaide, Australia. Aust. Soc. Anim. Prod., Wagga Wagga, Australia.

Grondahl-Nielsen, C., H. B. Simonsen, J. D. Lund, and M. Hesselholt. 1999. Behavioral, endocrine and cardiac responses in young calves undergoing dehorning without and with use of sedation and analgesia. Vet. J. 158:14-20.

Heinrich, A., T. F. Duffield, K. D. Lissemore, and S. T. Millman. 2010. The effect of meloxicam on behavior and pain sensitivity of dairy calves following cautery dehorning with a local anesthetic. J. Dairy Sci. 93:2450-2457.

Heinrich, A., T. F. Duffield, K. D. Lissemore, E. J. Squires, and S. T. Millman. 2009. The impact of meloxicam on postsurgical stress associated with cautery dehorning. J. Dairy Sci. 92:540-547.

Hempstead, M. N., J. R. Waas, M. Stewart, V. M. Cave, and M. A. Sutherland. 2017. Behavioral response to cautery disbudding in dairy goat kids. Appl. Anim. Behav. Sci. 194:42-47.
Ingvast-Larsson, C., M. Hogberg, U. Mengistu, L. Olsen, U. Bondesson, and K. Olsson. 2011. Pharmacokinetics of meloxicam in adult goats and its analgesic effect in disbudded kids. J. Vet. Pharmacol. Ther. 34:64-69.

Irvine, C. H., and S. L. Alexander. 1994. Factors affecting the circadian rhythm in plasma cortisol concentrations in the horse. Domest. Anim. Endocrinol. 11:227-238.

Karademir, U., H. Erdogan, M. Boyacioglu, C. Kum, S. Sekkin, and M. Bilgen. 2016. Pharmacokinetics of meloxicam in adult goats: a comparative study of subcutaneous, oral and intravenous administration. N. Z. Vet. J. 64:165-168.

Loretz, C., B. Wechsler, R. Hauser, and P. Rusch. 2004. A comparison of space requirements of horned and hornless goats at the feed barrier and in the lying area. Appl. Anim. Behav. Sci. 87:275-283.

McEwen, M. M., R. D. Gleed, J. W. Ludders, T. Stokol, F. Del Piero, and H. N. Erb. 2000. Hepatic effects of halothane and isoflurane anesthesia in goats. J. Am. Vet. Med. Assoc. 217:1697-1700.

Milligan, B. N., T. Duffield, and K. Lissemore. 2004. The utility of ketoprofen for alleviating pain following dehorning in young dairy calves. Can. Vet. J. 45:140-143.

Morisse, J. P., J. P. Cotte, and D. Huonnic. 1995. Effect of dehorning on behavior and plasma-cortisol responses in young calves. Appl. Anim. Behav. Sci. 43:239-247.

Nfor, O. N., J. P. W. Chan, M. Kere, and H. C. Peh. 2016. Disbudding pain: The benefits of disbudding goat kids with dexmedetomidine hydrochloride. Small Rumin. Res. 139:60-66.

Offinger, J., H. Meyer, J. Fischer, S. B. Kastner, M. Piechotta, and J. Rehage. 2012. Comparison of isoflurane inhalation anaesthesia, injection anaesthesia and high volume caudal epidural anaesthesia for umbilical surgery in calves; metabolic, endocrine and cardiopulmonary effects. Vet. Anaesth. Analg. 39:123-136.

Patt, A., L. Gygax, B. Wechsler, E. Hillmann, R. Palme, and N. M. Keil. 2012. The introduction of individual goats into small established groups has serious negative effects on the introduced goat but not on resident goats. Appl. Anim. Behav. Sci. 138:47-59.

Prunier, A., A. M. Mounier, and M. Hay. 2005. Effects of castration, tooth resection, or tail docking on plasma metabolites and stress hormones in young pigs. J. Anim. Sci. 83:216-222.

Smith, M. C., and D. M. Sherman. 2009a. Anesthesia. Pages 710-720 in Goat Medicine. 2nd ed. Wiley-Blackwell, Ames, IA.

Smith, M. C., and D. M. Sherman. 2009b. Dehorning and descenting. Pages 723-731 in Goat Medicine. 2nd ed. Wiley-Blackwell, Ames, IA.

Smith, M. C., and D. M. Sherman. 2009c. Fundamentals of goat practice. Pages 3-20 in Goat Medicine. 2nd ed. Wiley-Blackwell, Ames, IA.

Sutherland, M. A., G. L. Lowe, F. J. Huddart, and M. Stewart. 2016. An investigation of automated measures for assessing pain-induced distress in dairy calves. Pages 103-105 in Proc. N. Z. Soc Anim Prod., Adelaide, Australia. N. Z. Soc. Anim. Prod., Cambridge, New Zealand.

Thompson, K. G., R. S. Bateman, and P. J. Morris. 2005. Cerebral infarction and meningoencephalitis following hot-iron disbudding of goat kids. N. Z. Vet. J. 53:368-370.

Willett, L. B., and R. E. Erb. 1972. Short-term changes in plasma corticoids in dairy-cattle. J. Anim. Sci. 34:103-111.

Wright, H. J., D. S. Adams, and F. J. Trigo. 1983. Meningoencephalitis after hot-iron disbudding of goat kids. Vet. Med. Small Anim. Clin. 78:599-601.

Zobel, G., D. M. Weary, K. Leslie, N. Chapinal, and M. A. von Keyserlingk. 2015. Technical note: validation of data loggers for recording lying behavior in dairy goats. J. Dairy Sci. 98:1082-1089. 
APPENDIX

Table A1. Comparison of ethograms from Hempstead et al. (2017) and the current paper

Description

Behavior Hempstead et al. (2017)

Head shaking A rapid tilting of the head from side to side a minimum of three times concluding with a return to neutral position. Head shakes separated by $>1 \mathrm{~s}$ are considered separate events.

Head scratching

Tilting of the head so the rear foot scratches any part of the head (excluding neck). The rear fetlock must reach the shoulder and includes attempts (i.e., the foot does not contact the head but is raised off the ground). Head scratches separated by $>1 \mathrm{~s}$ are considered separate events.

Self-grooming

The kid's mouth contacts any part of the body or legs. A separate self-grooming event is considered to occur after a pause of $>1 \mathrm{~s}$.

Feeding

The mouth covers at least half of the nipple of the feeding bucket for $>3 \mathrm{~s}$, usually followed by suckling motions.

Repetitions following separation of the mouth from the nipple of $>3 \mathrm{~s}$ are considered separate events.
Current paper

Rapid continuous tilting of the head from side to side concluding with a return to neutral position. Head shakes separated by $>1 \mathrm{~s}$ are considered separate events.

The rear foot touches any part of the head or neck (including collar). Scratches separated by $>1$ s were considered separate events.

The kid's muzzle contacts any part of the body or legs (excluding hoof) with a rhythmic back and forth motion. A separate grooming event was considered to occur after a pause of $>1 \mathrm{~s}$.

Day: The mouth covers at least half of the nipple of the feeding bucket for $>3 \mathrm{~s}$, usually followed by suckling motions. Repetitions following separation of the mouth from the nipple of $>3 \mathrm{~s}$ were considered separate events. Night: The body is positioned facing the feeder and the muzzle is within a head's length of the nipple. A separate visit was considered to occur after the front legs moved away from the feed bucket. 